\title{
Virtual sensors for 2D vector field tomography
}

\author{
Archontis Giannakidis, ${ }^{1}$ Leonidas Kotoulas, ${ }^{2}$ and Maria Petrou ${ }^{2, *}$ \\ ${ }^{1}$ Faculty of Engineering and Physical Sciences, University of Surrey, Guildford GU2 7XH, UK \\ ${ }^{2}$ Department of Electrical and Electronic Engineering, Imperial College, Exhibition Road, \\ South Kensington, London SW7 2AZ, UK \\ *Corresponding author: maria.petrou@imperial.ac.uk
}

Received May 26, 2009; revised January 28, 2010; accepted February 10, 2010; posted March 29, 2010 (Doc. ID 111804); published May 14, 2010

\begin{abstract}
We consider the application of tomography to the reconstruction of 2-D vector fields. The most convenient sensor configuration in such problems is the regular positioning along the domain boundary. However, the most accurate reconstructions are obtained by sampling uniformly the Radon parameter domain rather than the border of the reconstruction domain. This dictates a prohibitively large number of sensors and impractical sensor positioning. In this paper, we propose uniform placement of the sensors along the boundary of the reconstruction domain and interpolation of the measurements for the positions that correspond to uniform sampling in the Radon domain. We demonstrate that when the cubic spline interpolation method is used, a 60 times reduction in the number of sensors may be achieved with only about $10 \%$ increase in the error with which the vector field is estimated. The reconstruction error by using the same sensors and ignoring the necessity of uniform sampling in the Radon domain is in fact higher by about $30 \%$. The effects of noise are also examined. (C) 2010 Optical Society of America

OCIS codes: $110.0110,110.6955,110.6960,100.3190$.
\end{abstract}

\section{INTRODUCTION}

The vectorial Radon transform of a 2 -D vector field arises naturally in applications the measurements of which are inherently line integrals of the inner product of the investigated vector field with a fixed vector. The reconstruction of the examined vector field, by means of inverting the vectorial Radon transform, allows one to determine the internal structure of a system without physically probing the interior. Typical examples of vector fields that can be recovered include blood flow in vessels [1,2]; oceanic fluid mesoscale velocity [3-5]; fluid flow [6-13]; electric field in Kerr materials [14-16]; the gradient of the refractive index field [11]; velocity of heavy particles [17]; density in supersonic expansions and flames [18]; non-destructive stress distribution of transparent specimens [19,20]; temperature distributions and velocity vector fields in furnaces [21]; and magnetic fields in tokomaks [22].

The problem of reconstructing a $2-\mathrm{D}$ vector field in the continuous domain from line-integral measurements is underdetermined $[10,11,19,23]$. In particular, it has been shown that only one component of the vector field can be recovered from the tomographic measurements. The recovered component is either the curl-free (irrotational) part or the divergence-free (solenoidal) part, depending on the physical principle of the measurements, namely the relation between the obtained set of measurements and the investigated vector field.

However, it has been recently demonstrated [24] that in the discrete domain, the problem is tractable. In particular, it was shown that one may estimate both components of a $2-D$ vector field at a finite number of points of a domain by simply using the integrals of the projections of the field along lines tracing the domain [24]. This is possible of course, under the assumption that the density of the sampling points does not violate the Nyquist limit of signal sampling, i.e. that the field is band-limited and it does not contain frequencies that are higher than half the sampling frequency. Cases which cannot be dealt with by this approach are fields that contain singularities, as singularities make the field to be not band-limited. With the assumption of a band-limited field, the solution of the problem is always possible, as for a sampling grid of $N$ $\times N$, one has $N^{2}$ unknowns, and $6 N^{2}$ measurements, considering all pairs of points on the perimeter of the grid, when the paired points do not belong to the same side of the perimeter.

The reconstruction method described in [24] is based on linear algebra. It formulates the tomographic vector field reconstruction problem in terms of a system of linear equations. This matrix formalism is analogous to the Radon transform scheme. Hence, solving the system of linear equations that was obtained in [24] is equivalent to inverting the vectorial Radon transform. According to the theory of the Radon transform [25], a necessary requirement for producing accurate reconstructions is to sample uniformly the Radon domain parameter space, defined by the length of the normal to a tracing line, $\rho$, and the angle this normal forms with the positive $x$ axis, $\theta$ (see Fig. 1). However, sampling this space uniformly has two major drawbacks:

(i) the required sensors are not placed uniformly in the $(x, y)$ Cartesian coordinates of the domain;

(ii) the sensors that have to be placed at the ends of a line tracing the domain may be impractically close to the sensors of another tracing line, as dictated by the uniform sampling of the $(\rho, \theta)$ space.

In this paper, we show how these problems may be 


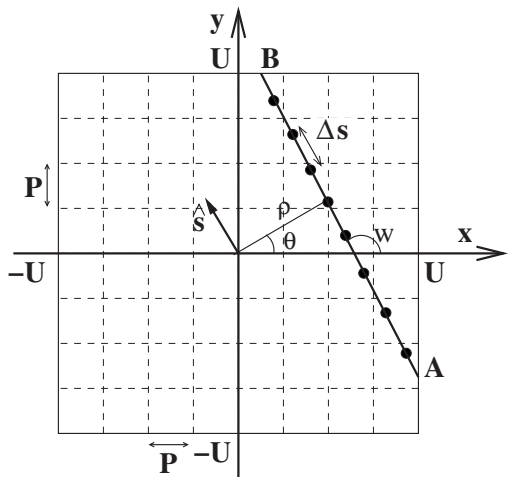

Fig. 1. Tracing line $A B$ unites two virtual sensors that reside at points $A$ and $B$. The tracing line is defined by the two parameters $\rho$ and $\theta$ (Radon domain coordinates) and goes through the digitized square reconstruction region of size $2 U \times 2 U$. The line segment is sampled with sampling step $\Delta s$. The angle between the line segment and the positive direction of the $x$-axis is $w$. The size of the tiles with which we sample the 2 -D space is $P \times P$. Also shown is the unit vector $\hat{\mathbf{s}}$, which is parallel to line segment $A B$.

overcome by using virtual sensors. The data values at these sensors that correspond to uniform sampling in the $(\rho, \theta)$ domain are obtained from the known values of the true sensors that are placed at regular points in relation to the Cartesian coordinates $(x, y)$ by using interpolation. This approach allows one to use as many tracing lines as one can afford, taking into consideration the computational cost of solving the corresponding system of linear equations. However, the increase of the number of the available line-integral data in such a way is not limited by physical constraints on sensor placement.

This paper is organized as follows. In Section 2 we formulate the problem, set up our notation and give a brief summary of the algebraic reconstruction method presented in [24]. In Section 3 we present an example of static electric field reconstruction and demonstrate the effect of the use of interpolated data on the quality of reconstruction. In Section 4 we examine the effect of the employment of interpolated measurements on resilience to noise. We conclude in Section 5.

\section{RECONSTRUCTION METHODOLOGY}

The treatment in this study is similar to the one in [24]. We perform the analysis in the digital domain. Let us assume that we have the digitized square 2-D domain that is shown in Fig. 1, within which we want to recover vector field $\mathbf{f}(x, y)=f_{x}(x, y) \hat{\mathbf{x}}+f_{y}(x, y) \hat{\mathbf{y}}$. The length of each side of the square domain is taken to be equal to $2 U$, and the origin of the axes of the coordinate system is chosen to be at the center of the domain. The square domain is divided into tiles of finite size, $P \times P$, so that $2 U / P$ is an integer. The goal is to recover vector field $\mathbf{f}(x, y)$ at the center of every tile of this space, namely the sampling points of the domain.

Moreover, we assume, in line with [24], that ideal point sensors that integrate only the component of the field projected on the line reside on predetermined and regularly placed positions of the whole border of the 2-D square domain. These positions are the middle points of the bound- ary edges of all boundary tiles. However, in order to achieve the best vector field reconstruction, the data should not be collected by these regularly placed sensors, but by sensors that correspond to uniform sampling of parameters $\rho$ and $\theta$. Therefore, we propose to use interpolated measurements that correspond to uniform sampling of the $(\rho, \theta)$ space.

Let us consider a tracing line $A B$ (see Fig. 1) that connects two sensors. In terms of parameters $\rho$ and $\theta$, the equation of the line is

$$
\rho=x \cos \theta+y \sin \theta .
$$

Sampling $\rho$ and $\theta$ parameters uniformly results in a set of such lines. Each tracing line $(\rho, \theta)$ of the set yields a lineintegral measurement of the projection of the vector field along the line's direction. Since we assumed that each pair of sensors measures only the integral of the component of the vector field along the scanning line $(\rho, \theta)$, the integral transform that models the process of data acquisition is given by

$$
J_{1}=\int_{A B} \overline{\mathbf{f}}(x, y) \cdot \hat{\mathbf{s}} \mathrm{d} s=\int_{A B} f_{\|} \mathrm{d} s .
$$

Here $\hat{\mathbf{s}}=\cos w \hat{\mathbf{x}}+\sin w \hat{\mathbf{y}}$ is the unit vector along the integration (measurement) line $A B$ and $w$ is the angle between the tracing line and the positive direction of the $x$-axis (see Fig. 1). In addition, $\mathrm{d} s$ is an element of path length along this line, $\cdot$ is the symbol for the dot product of two vectors, and $f_{\|}$is the component of $\mathbf{f}(x, y)$ along $A B$. In order to translate into the digital domain, the integration expressed by Eq. (2) in the continuous domain the integral of the vector field along the tracing line has to be expressed in terms of the components of the field at the sampling points of the 2-D grid. To do that, the tracing line is sampled with step $\Delta s$ (see Fig. 1), and the value of the vector field assigned to each center of segment of length $\Delta s$ is the unknown value of the field at the nearest sampling point of the reconstruction domain. Along each segment of length $\Delta s$, the vector field is assumed constant, equal to the assigned value of the vector field at the corresponding sampling point of the line. It is possible, then, to approximate the integral of Eq. (2) by a sum, by projecting the value of the field at each sampling point $l$ of the line onto the vector that represents the direction of the line:

$$
J_{i}=\sum_{l} \overline{\mathbf{f}}_{l} \cdot \overline{\Delta \mathbf{s}}
$$

Here $\mathbf{f}_{l}=\left(f_{x l}, f_{y l}\right)$ are the unknown vector field values at sampling points $l$ and $\overline{\Delta \mathbf{s}}=\Delta s \hat{\mathbf{s}}$. The number of Eqs. (3) we have depends on the number of tracing lines between the virtual sensors we consider. In general, it is an overdetermined system of linear equations, and its solution is obtained in the least-square error sense.

To summarize, our formulation of the vector field reconstruction problem may be written in matrix form as

$$
\mathbf{C} \overline{\mathbf{g}}=\overline{\mathbf{b}}
$$

where $\mathbf{b} \in \mathbb{R}^{C_{r} \times 1}$ is the vector that contains the projection measurements between virtual sensors wrapped into a 
vector, $\mathbf{g} \in \mathrm{R}^{C_{c} \times 1}$ is the set of the components of the vector field to be reconstructed at all sampling points of the 2-D digitized domain written as a vector, and $\mathbf{C} \in \mathrm{R}^{C_{r} \times C_{c}}$ is the system matrix containing the weight factors between each of the components of the vector field at every reconstruction point and each of the corresponding tracing line orientations from the set of measurements. System matrix $\mathbf{C}$ is obtained from the analysis described in [24]. The solution of the overdetermined system (4) that minimizes the norm of the residual vector is obtained by solving the system of equations

$$
\mathbf{C}^{T} \mathbf{C} \overline{\mathbf{g}}=\mathbf{C}^{T} \overline{\mathbf{b}}
$$

where $\mathbf{C}^{T}$ denotes the transpose of $\mathbf{C}$ and the matrix of coefficients $\mathbf{C}^{T} \mathbf{C}$ is square. Hence, the least-square error solution (or else the vector field reconstruction result) is given by

$$
\overline{\mathbf{g}}=\left(\mathbf{C}^{T} \mathbf{C}\right)^{-1} \mathbf{C}^{T} \overline{\mathbf{b}} .
$$

In the next section we demonstrate that the reconstruction results we obtain using interpolated line-integral measurements observed at virtual sensors as described above are more accurate than the ones obtained in [24], where reconstruction was based on line-integral measurements collected by sensors that resided at regular points in relation to the Cartesian coordinates $(x, y)$.

\section{EXAMPLE: ELECTRIC FIELD IMAGING}

We consider the case where the vector field that we want to recover is the electric field created by a static charge. There are many ways to recover the electric field from boundary data. However, here we use the electric field only to demonstrate our method. In order to avoid problems with singularities, we place the source of the vector field outside the bounded 2-D area. In a real physical system, we do not expect to have to deal with real singularities anyway. We would like to stress that the problem we solve is intentionally kept simple in order to demonstrate the method. So, instead of avoiding singularities by using a realistic version of Coulomb's law for sources of finite size, we place the source outside the domain of interest and make it infinitesimally small.

For a static electric field, every voltage difference between any two points is the line-integral of the field projected along the line that connects these two points. Therefore, we assume that the boundary sensors measure the potential, so that the difference in the measurements between any two such sensors gives the vectorial Radon transform of the electric field. For the simulations we present here, the potential in all these sensors is obtained by using Coulomb's law. It must be noted that the electric field is irrotational, so according to [10], only transversal measurements would be helpful to recover this field. However, the only realizable measurements for this application are longitudinal.

For our experiments, we employed the digital square reconstruction domain of Fig. 1 and chose $2 U=11$ as domain size and $P=1$ as tile size. Hence, the domain consisted of 121 tiles and the number of the unknowns (the $E_{x}$ and $E_{y}$ components of the field at the center of every tile of the domain) was 242 . In addition, the line segments joining sensors were sampled with a step equal to $1(\Delta s$ $=1$ ). Four different cases for the location of the source of the vector field are reported. To exemplify the theory of the study described in this paper, we performed five sets of experiments for each source location.

The first set of experiments was performed following the analysis described in [24]. Hence, we considered the practical case where ideal point sensors are regularly placed (RS) in relation to the Cartesian coordinates $(x, y)$ in known and predetermined positions of the whole border of the domain. These positions were the middle points of the boundary edges of all boundary tiles. Therefore, we used 11 sensors in every side of the boundary of the square domain. We considered all possible voltage differences between pairs of these sensors, apart from sensors lying on the same border line, and we formed the system of linear equations according to the description of [24]. The number of linear equations was 726 .

In the second set of experiments, we used the same sensor placement as in the first set of simulations. However, we performed the vector field reconstruction by relying only on interpolated line-integral data observed at virtual sensors that corresponded to uniform sampling of the $(\rho, \theta)$ Radon space, as proposed in this paper. To obtain the positions where the virtual sensors had to be inserted, we considered for the Radon domain parameters the sampling steps recommended in [26,27]: $\Delta \rho=1$ and $\Delta \theta=3^{\circ}$. The data values of the virtual sensors were obtained from the data of the true sensors that were regularly placed in relation to $(x, y)$ by using some interpolation method. In this study we examined the following methods: 1-D linear interpolation (IP1) [28], 1-D piecewise cubic spline interpolation (IP2) [28], 1-D piecewise cubic Hermite interpolation (IP3) [28], bilinear interpolation (IP4) [28], bicubic interpolation (IP5) [28] and 2-D spline interpolation (IP6) [28] (see Appendix A). The selected sampling steps of parameters $\rho$ and $\theta$ resulted in having 6 samples for the radial parameter and 120 samples for the angular parameter so that the region of interest (Fig. 1) was fully covered. Consequently, the overdetermined system of linear equations, the solution of which gave the reconstructed field, had $720(=6 \times 120)$ equations, almost the same number as in the first set of experiments.

In the third set of experiments, we used uniform sampling (US) in the parameter space, the same as in the second set of experiments. However, the sensor placement was different. In particular, the vector field recovery was not based on interpolated measurements, but we assumed that there are sensors at the ends of all lines that trace the domain and that are uniformly distributed in the $(\rho, \theta)$ space.

In the fourth and fifth sets of experiments, the vector field reconstruction was performed as in the second and third sets of experiments, respectively, apart from the fact that the employed sampling rates were increased twofold: $\Delta \rho=0.5$ and $\Delta \theta=1.5^{\circ}$. This resulted in having $2640(=11$ $\times 240$ ) linear equations. We must note that for the case where interpolated measurements are used for the reconstruction, such an increase of line-integral data is not limited by the physical limitations that the sensor placement imposes. In addition, this increase was made taking into 
consideration that the resulting system of equations would not be prohibitively large and its solution would not increase the processing time significantly.

The reconstruction results, namely the solution of the overdetermined systems of linear equations for the five sets of experiments and the four source locations were obtained by applying the least-squares method. The Householder orthogonalization method [29], which is a numerically useful procedure in order to solve mean square value problems for cases where the condition number of the matrix of coefficients is large [30], was also tested for our reconstruction problem. However, the results we obtained were identical with the results we obtained using the least-squares method. Moreover, it must be noted that since the residual we computed by using the least-squares method was not large when compared with the solution vector, there was no need to use the Cholesky method [31].

The relative magnitude reconstruction error values (i.e., the absolute values of the differences between the magnitudes of the reconstructed fields and the theoretical ones as obtained by using directly the governing Coulomb's law divided by the theoretical magnitude) and the absolute angular reconstruction error values (i.e., the absolute angular differences (in degrees) between the reconstructed vector field values and the theoretical ones) for the five sets of experiments and for the four locations of the source were calculated. The means of these errors per reconstruction tile are shown in Figs. 2-5.

We note from these figures that the cases where we used interpolated measurements obtained at virtual sensors that correspond to uniform sampling in the $(\rho, \theta)$ space outperform the case where reconstruction was based on line-integral data obtained at sensors that are regularly placed in relation to the $(x, y)$ coordinates. In addition, the higher the sampling rate of parameters $\rho$ and $\theta$, the more accurate the obtained reconstruction. By careful inspection of Figs. 2-5, we may also see that the interpolation method that led to the most accurate reconstruction was the 1-D piecewise cubic spline interpolation $[28,32,33]$. In particular, it was found that the average difference in vector field orientation measured in degrees was $34 \%$ lower when we employed interpolated data (using the 1-D piecewise cubic spline method) that correspond to uniform sampling in the Radon domain with $\Delta \rho=0.5$ and $\Delta \theta=1.5^{\circ}$, as opposed to the regular positioning of sensors in the $(x, y)$ domain, while the average error in magnitude was lower by $30 \%$. The reconstructed vector fields for the case where we used interpolated data (1-D piecewise cubic spline method) that correspond to uniform sampling in the Radon domain with $\Delta \rho=0.5$ and $\Delta \theta$ $=1.5^{\circ}$ are shown in Fig. 6(a). For the sake of comparison, Fig. 6(b) depicts also the respective theoretical electric fields that were obtained by using directly the governing Coulomb's law.

From Figs. 2-5, we can also see that, as expected, when uniform sampling of the parameter space is used, the use of actual measurements results in more accurate reconstructions than when interpolated measurements are used. In particular, it was found that for sampling steps $\Delta \rho=0.5$ and $\Delta \theta=1.5^{\circ}$, the case where actual measurements were used led to $8 \%$ and $14 \%$ lower angular and magnitude errors on average, respectively, as opposed to using interpolated measurements and the 1-D piecewise cubic spline method. However, by relying on interpolated measurements, the number of the overall sensors required is about 60 times lower than the respective number when actual measurements are used. Hence, the employment of interpolated measurements that is proposed in this paper results in a much more practical sensor configuration.

\section{VIRTUAL SENSORS AND NOISE}

An important issue when solving inverse problems is the sensitivity of the solution to noise. In this section we investigate the effects of noise on the use of interpolated measurements obtained at virtual sensors that correspond to uniform sampling of the $(\rho, \theta)$ space. In all experiments reported in Section 3 , the sensors were placed exactly at the positions we had decided, and the measurement taken by each sensor was exactly the value pre-
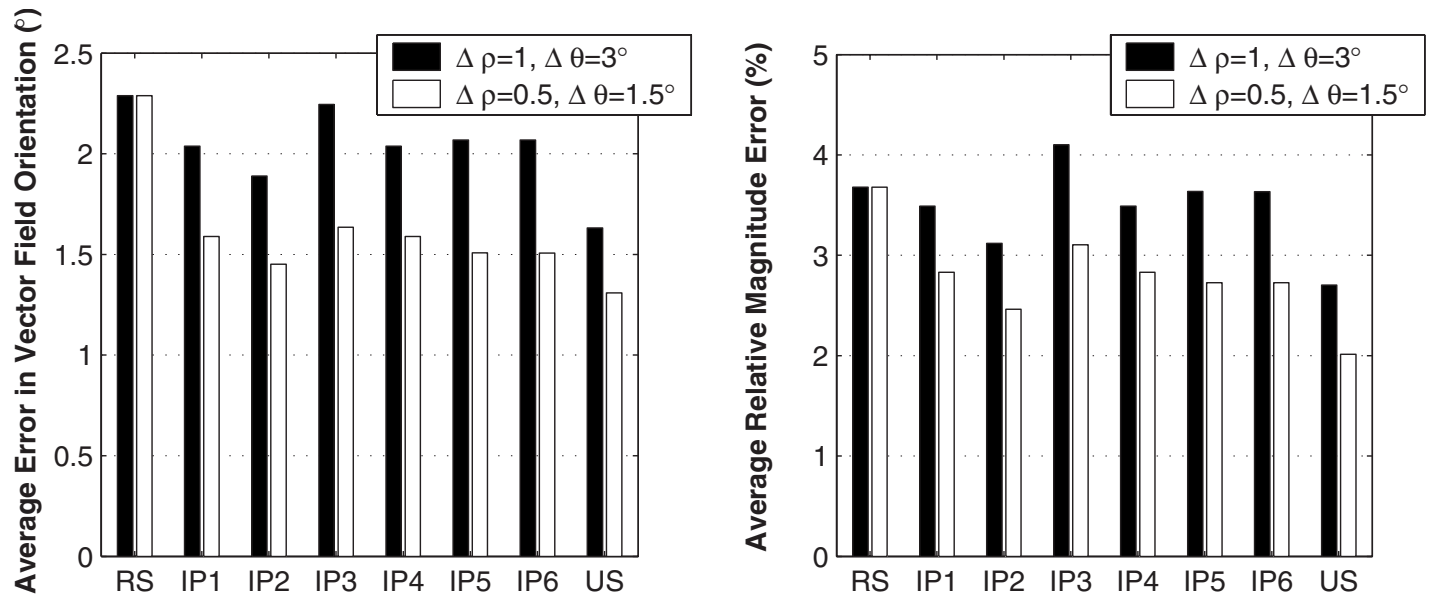

Fig. 2. Comparison of the reconstruction performance for the cases when reconstruction was based on: (i) line-integral data from regularly placed sensors (RS) in relation to $(x, y)$ coordinates; (ii) interpolated line-integral data obtained at virtual sensors that corresponded to uniform sampling of the Radon space and the employed interpolation method was the 1-D linear (IP1), the 1-D piecewise cubic spline (IP2), the piecewise cubic Hermite (IP3), the bilinear (IP4), the bicubic (IP5), and the 2-D spline (IP6); (iii) uniform sampling (US) of the parameter space using the actual measurements. The location of the source of the electric field was at $(19,-19)$. 

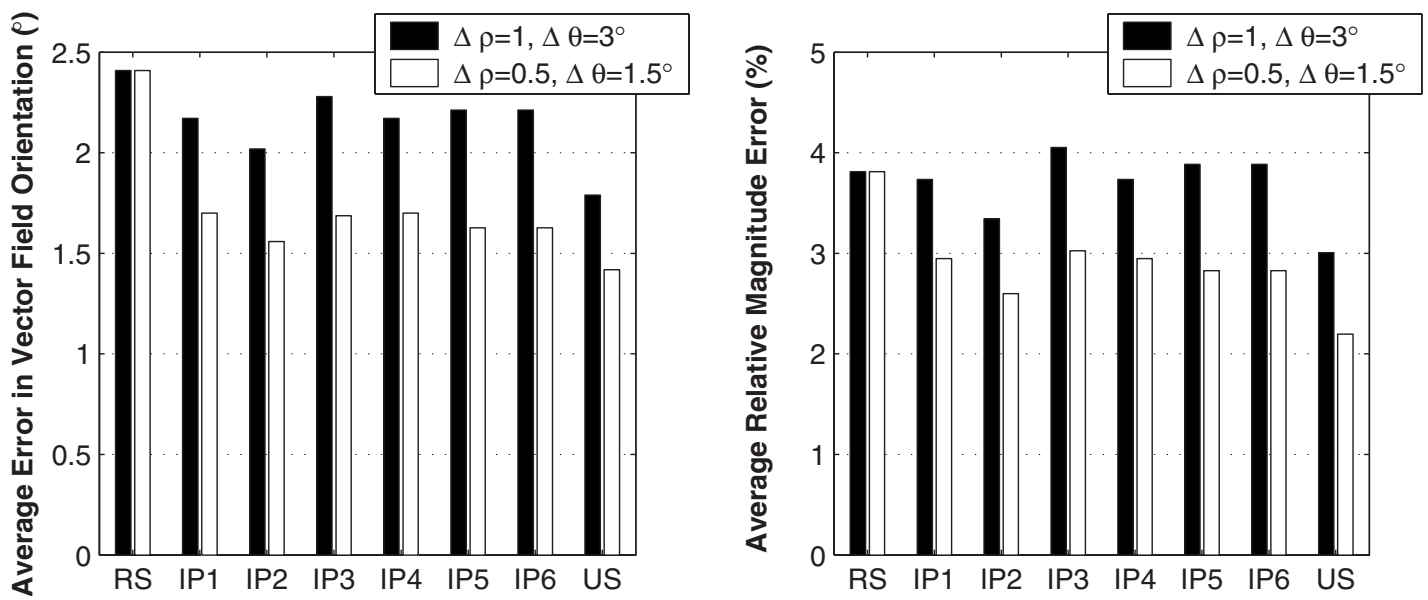

Fig. 3. As in Fig. 2, but here the location of the source of the electric field was at $(-16,21)$.

dicted by Coulomb's law. In a practical system, however, some of the sensor measurements are expected to have inaccuracies, and some of the sensor positions are also expected to be somehow inaccurate. To emulate these effects, we considered the following.

(i) A noise value was added to a measurement as a fraction of the true value, with random sign. For example,
$2 \%$ noise means that the sensor measurement was changed by $2 \%$ of the value dictated by Coulomb's law. The change was either incremental or decremental, the choice made at random for each sensor.

(ii) A sensor was moved away from its true position by a fraction of the true position. For example, if according to the theory, a sensor should be placed at position $(x, y)$, and we consider a $2 \%$ error, then the coordinates of this sensor
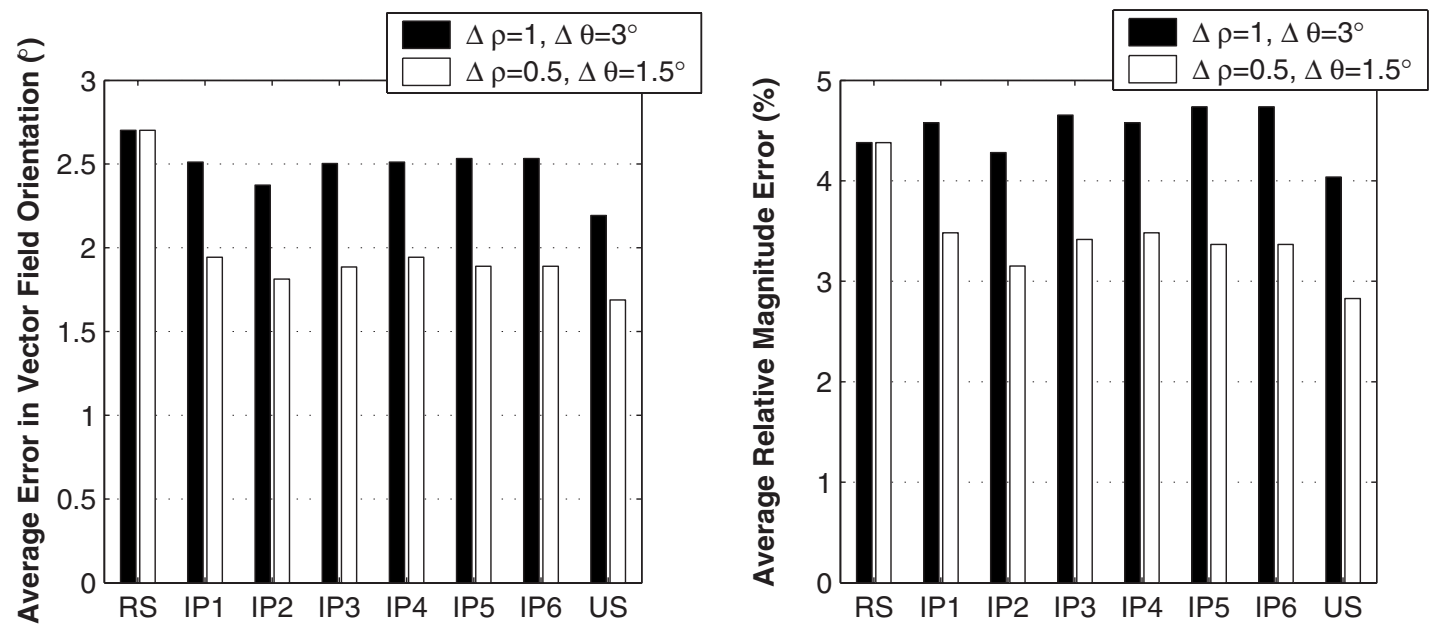

Fig. 4. As in Fig. 2, but here the location of the source of the electric field was at $(-21,-12)$.
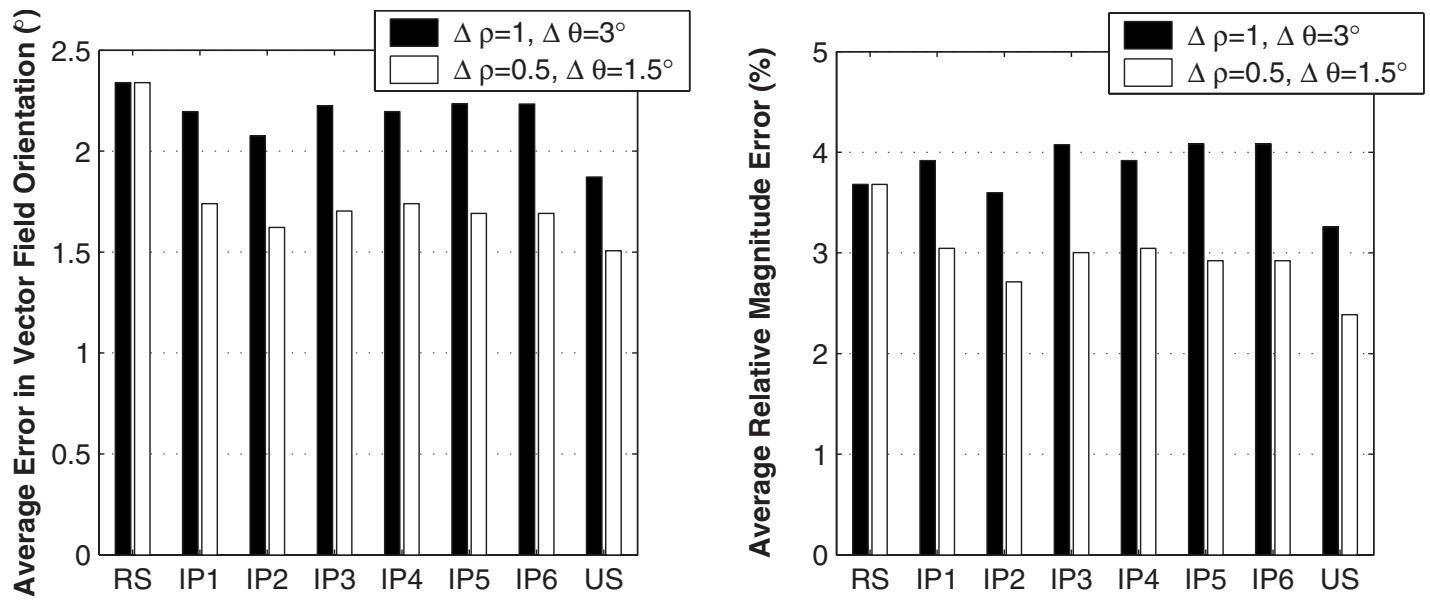

Fig. 5. As in Fig. 2, but here the location of the source of the electric field was at $(24,14.5)$. 
(a) Recovered Field
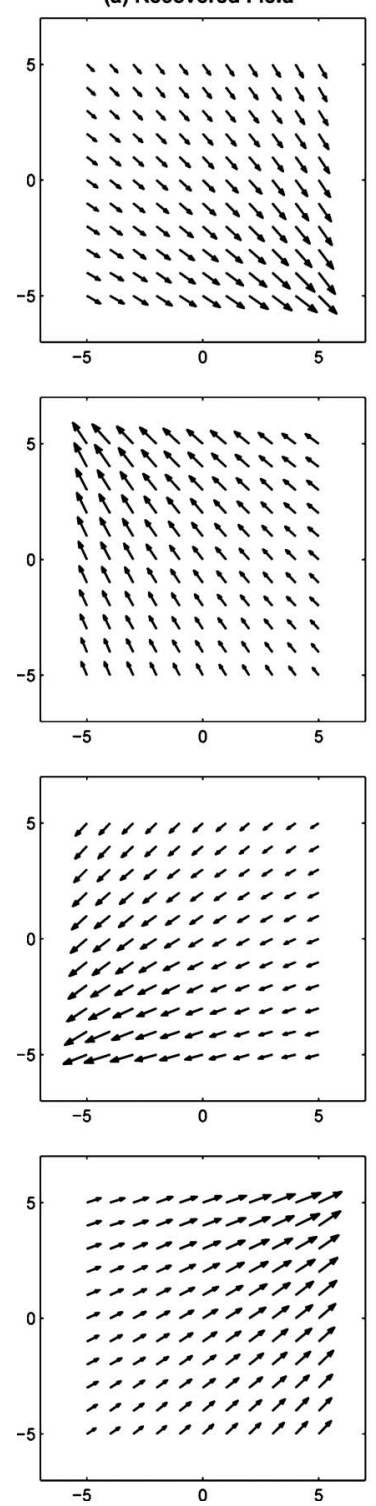

(b) Theoretical Field
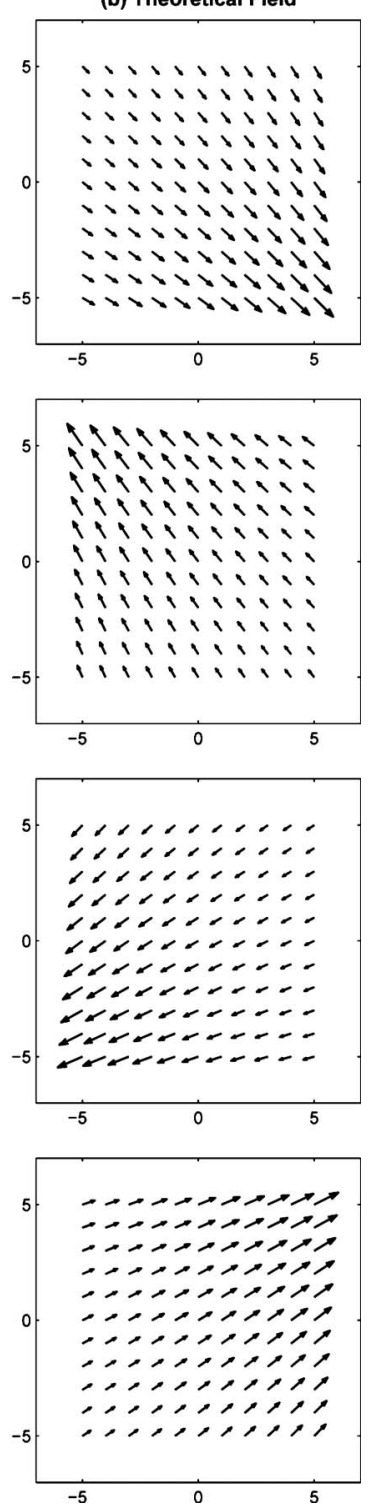

Fig. 6. Simulation results when the location of the source of the electric field was (from top to bottom) at $(19,-19),(-16,21)$, $(-21,-12)$, and $(24,14.5)$ : (a) the recovered vector field when reconstruction was based on interpolated line-integral data (1-D piecewise cubic spline method) obtained at virtual sensors that corresponded to uniform sampling of the Radon space with $\Delta \rho$ $=0.5$ and $\Delta \theta=1.5^{\circ} ;$ (b) the theoretical electric field as computed from Coulomb's law.

were shifted by $2 \%$ of the corresponding correct values, with a positive or negative sign chosen at random.

(iii) Both the above errors were considered simultaneously.

We performed four series of experiments by perturbing, by the three types of noise described above, (a) only $25 \%$ of the sensors, (b) $50 \%$ of the sensors, (c) $75 \%$ of the sensors, (d) all sensors. In order to evaluate the robustness of the employment of interpolated data as proposed in this study against noise, we examined for each series of experiments the following three cases: (a) when integral data from regularly placed sensors were used; (b) when interpolated measurements that correspond to uniform

sampling with $\Delta \rho=0.5$ and $\Delta \theta=1.5^{\circ}$ were used; and (c) when actual measurements that correspond to uniform sampling with $\Delta \rho=0.5$ and $\Delta \theta=1.5^{\circ}$ were used. For every noise value (of each noise type, each reconstruction approach, and each percentage of perturbed sensors), fifty simulations were performed and the average reconstruction errors in relative magnitude and absolute vector field orientation were obtained. The source for all the simulations was located at $(19,-19)$.

The results of these experiments are shown in Figs. $7-10$. We observe that the employment of interpolated measurements observed at virtual sensors that correspond to uniform sampling of the $(\rho, \theta)$ space that is proposed in this paper increases the resilience to all three types of noise, when compared with the case of the sensor regular positioning in the $(x, y)$ domain proposed in [24].

The assumed noise model in the above experiments was signal dependent. Noise processes of this type are inherent in many fields such as optics [34], kinematics [35], and magnetic resonance imaging [36]. Nevertheless, we also conducted experiments with fixed sensor positions and additive Gaussian noise of zero mean and sigma variance. In order to evaluate the robustness of the employment of interpolated data against noise, we examined the following three cases: (a) when integral data from regularly placed sensors were used; (b) when interpolated (1-D piecewise cubic spline) measurements that correspond to uniform sampling with $\Delta \rho=0.5$ and $\Delta \theta=1.5^{\circ}$ were used; and (c) when actual measurements that correspond to uniform sampling with $\Delta \rho=0.5$ and $\Delta \theta=1.5^{\circ}$ were used. We carried out simulations for the following values of SNR (in dB): 20, 25, 30, 35, 40, 45, 50, 55. The corresponding standard deviation of the noise was: $0.0383,0.0215$, $0.0121,0.0068,0.0038,0.0022,0.0012,0.0007$. For every noise (sigma) value, fifty simulations were performed and the average reconstruction errors in relative magnitude and absolute vector field orientation were obtained. The source for all the simulations was located at $(19,-19)$. The results of these experiments are shown in Fig. 11.

\section{DISCUSSION AND CONCLUSIONS}

The analysis of the vector field tomography problem in the continuous domain and the application of conventional (scalar) tomography theory leads to an underdetermined problem $[4,10,11]$. However, as was demonstrated in [24], the recovery of all components of a 2 -D vector field at the sampling points of a 2-D digitized bounded domain based only on a finite number of line-integral data may be achieved, assuming that the field is band-limited.

In this paper, we employ interpolated boundary data obtained at virtual sensors that correspond to uniform sampling of the $(\rho, \theta)$ space. The simulation results point out that this employment leads to a significant reduction of both the angular and magnitude reconstruction error as compared with the case where data from sensors that were regularly placed in relation to $(x, y)$ coordinates were used.

The employment of data that are collected at virtual sensors that correspond to uniform sampling in the $(\rho, \theta)$ domain allows us to use as many line-integral data as we can afford, taking into consideration the computational 


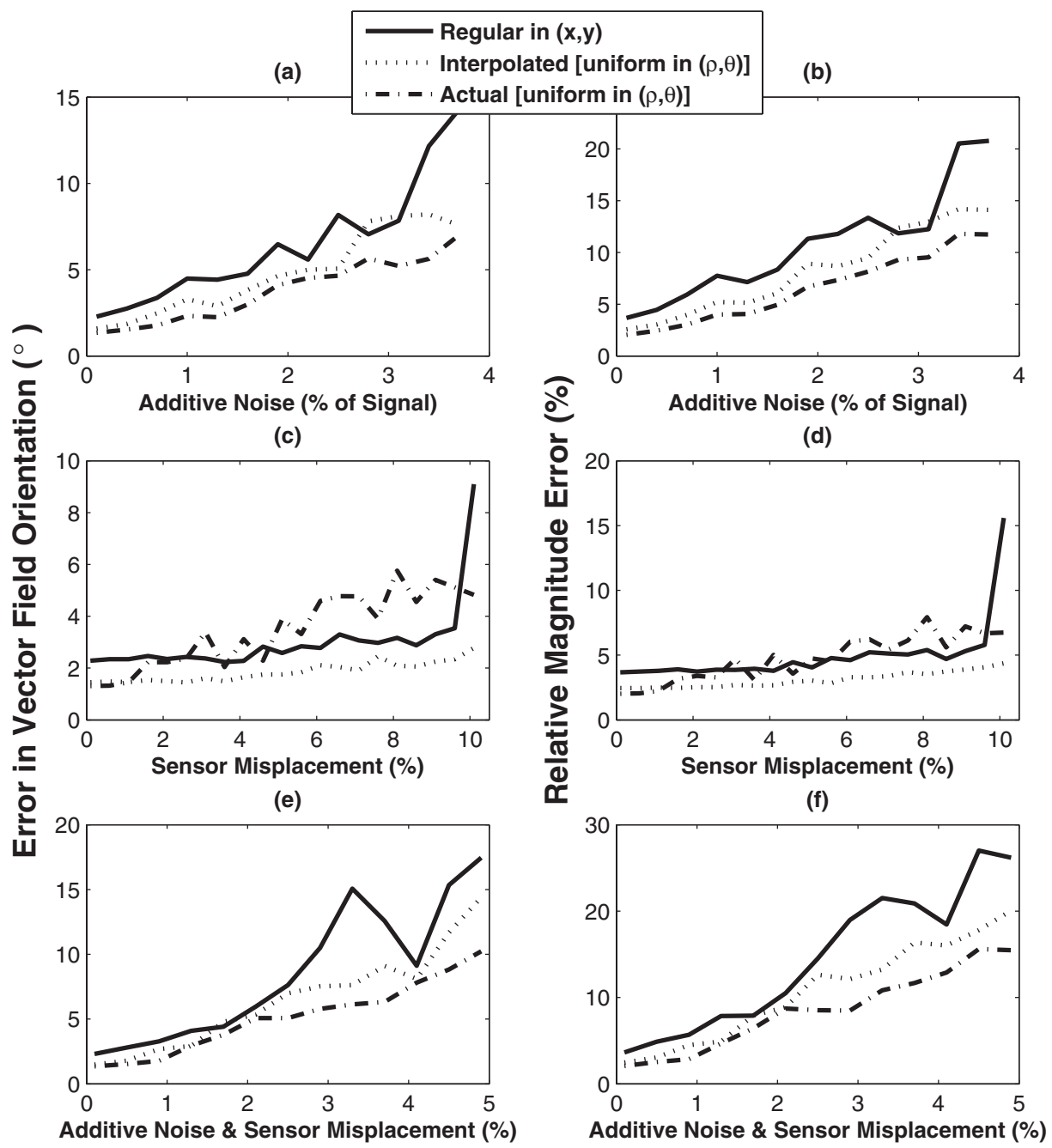

Fig. 7. Comparison of the reconstruction performance in noisy environments for the cases: (i) when integral data from regularly placed sensors were used; (ii) when interpolated measurements that corresponded to uniform sampling with $\Delta \rho=0.5$ and $\Delta \theta=1.5^{\circ}$ were used; (iii) when actual measurements that corresponded to uniform sampling with $\Delta \rho=0.5$ and $\Delta \theta=1.5^{\circ}$ were used. (a), (b) Errors in vector field orientation and magnitude when noise was added to the measurements of $25 \%$ of the sensors, as a percentage of the true value. (c), (d) Errors in vector field orientation and magnitude when small perturbations in the sensor positions were added. Position perturbations were a percentage of the true positions. (e), (f) Errors in vector field orientation and magnitude when both sensors' measurements and positions were changed by a percentage of their true values. In all cases, $25 \%$ of the sensors were perturbed.

cost of solving the corresponding system of linear equations. However, most importantly, the increase of the number of the available line-integral data in such a way is not limited by physical constraints on sensor placement. Hence, contrary to the case where the uniform sampling in the $(\rho, \theta)$ domain is combined with actual measurements, the employment of interpolated measurements as proposed in this paper achieves reconstruction of higher quality by maintaining at the same time a practical sensor configuration.

Another significant outcome of the study presented here is that the use of interpolated line-integral data obtained on virtual sensors results also in improved noise tolerance. This result is of great importance, especially in medical situations where dealing with noise is a major issue.

Part of the noise is introduced by the assumption that the value of the field remains constant along each segment of the tracing line. A more elaborate approach would consider the parts of each tracing line that belong to dif- ferent tiles with which we have covered the domain of the field and calculate the contribution of the field on the integral along each line taking that into consideration. This, however, would make the process far too complicated. We rely on the redundancy of the equations to reduce the error introduced this way.

The employment of interpolated data that correspond to uniform sampling of the $(\rho, \theta)$ space offers the possibility to increase the number of the available line-integral data without being limited by physical constraints on sensor placement or total scanning time. However, generating additional data points through interpolation does not increase the amount of available information. Hence, the amount of information contained in the set of interpolated data was the same as in the original case of [24]. The explanation for the results presented in this paper lies in Radon transform theory [25]. In particular, the method we developed is a direct algebraic reconstruction technique that performs inversion of the vectorial Radon transform. According to the theory of the Radon trans- 


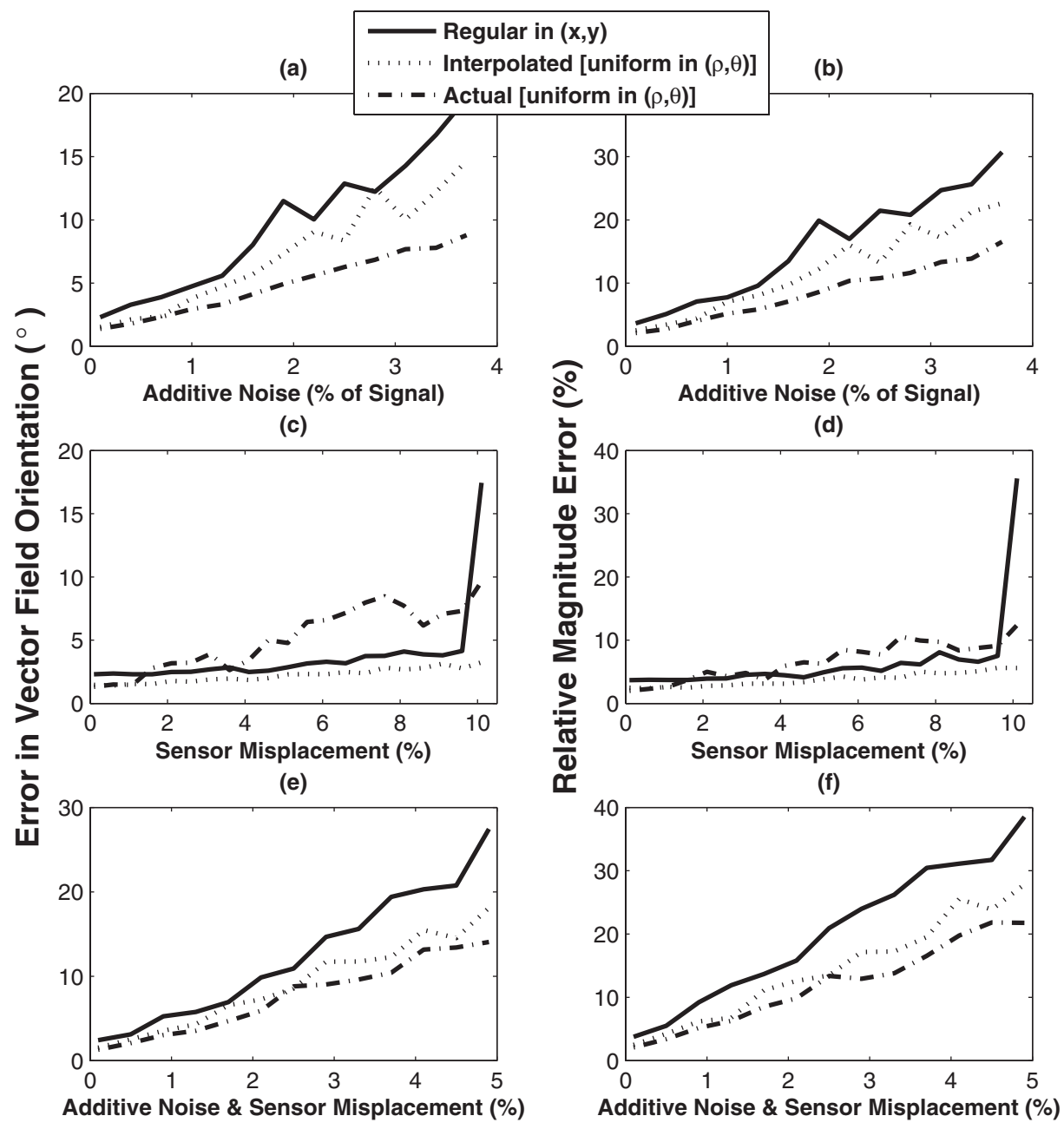

Fig. 8. As in Fig. 7, but 50\% of the sensors were perturbed.

form [25], a necessary requirement to produce reconstruction results of great accuracy when using discrete approximations is to have uniform distribution of projection data as functions of the two Radon domain variables normally designated as the radial and angular coordinates. Hence, employing interpolated data collected at virtual sensors that correspond to uniform sampling of the projection space results in feeding our reconstruction algorithm with data that are more favorable to reconstruction accuracy. However, it must be noted that increasing the number of interpolated measurements does not increase the accuracy of the reconstruction ad infinitum. Beyond a certain point, the benefit of achieving uniformity in the $(\rho, \theta)$ space is counterbalanced by the error of the numerical solution of the system of equations, as the measurements start becoming highly correlated and the coefficient matrix difficult to invert. Also, the time it takes to do the interpolation grows exponentially.

\section{APPENDIX A: INTERPOLATION METHODS}

Here we give the mathematics of the six interpolation methods used in this paper.

1. 1-D linear interpolation: Consider an unknown 1-D function $S(x)$. Also, assume the value of this function is known for $x=x_{A}$ and $x=x_{B}$. 1-D linear interpolation takes the two known values $S\left(x_{A}\right)$ and $S\left(x_{B}\right)$ and gives the interpolated value $S(x)$ at any point $x$ that lies in the interval $\left(x_{A}, x_{B}\right)$ by using the formula

$$
S(x)=S\left(x_{A}\right)+\left(x-x_{A}\right) \frac{\left(S\left(x_{B}\right)-S\left(x_{A}\right)\right)}{\left(x_{B}-x_{A}\right)} .
$$

2. 1-D piecewise cubic spline interpolation: Assume there is a set of $N$ data points $\left(x_{i}, S_{i}\right)$ $\left(i=1, \ldots, N ; x_{1}<x_{2}<\ldots x_{N}\right)$. The approximation interval $\left[x_{1}, x_{N}\right]$ is divided into $N-1$ subintervals by the so-called $N$ nodes $\left(x_{i}\right)$. In any subinterval $\left[x_{i}, x_{i+1}\right](i=1, \ldots, N-1)$, the interpolated value at a point $x$ that lies in this interval is given by

$$
S(x)=S_{i}(x)=a_{i}+b_{i}\left(x-x_{i}\right)+c_{i}\left(x-x_{i}\right)^{2}+d_{i}\left(x-x_{i}\right)^{3},
$$

where the spline coefficients are determined by the given set of data and the conditions of continuity of $S(x), S^{\prime}(x)$, and $S^{\prime \prime}(x)$ at the interior nodes [31]. Hence, the cubic spline consists of $N-1$ piecewise cubic polynomials between the data points. 


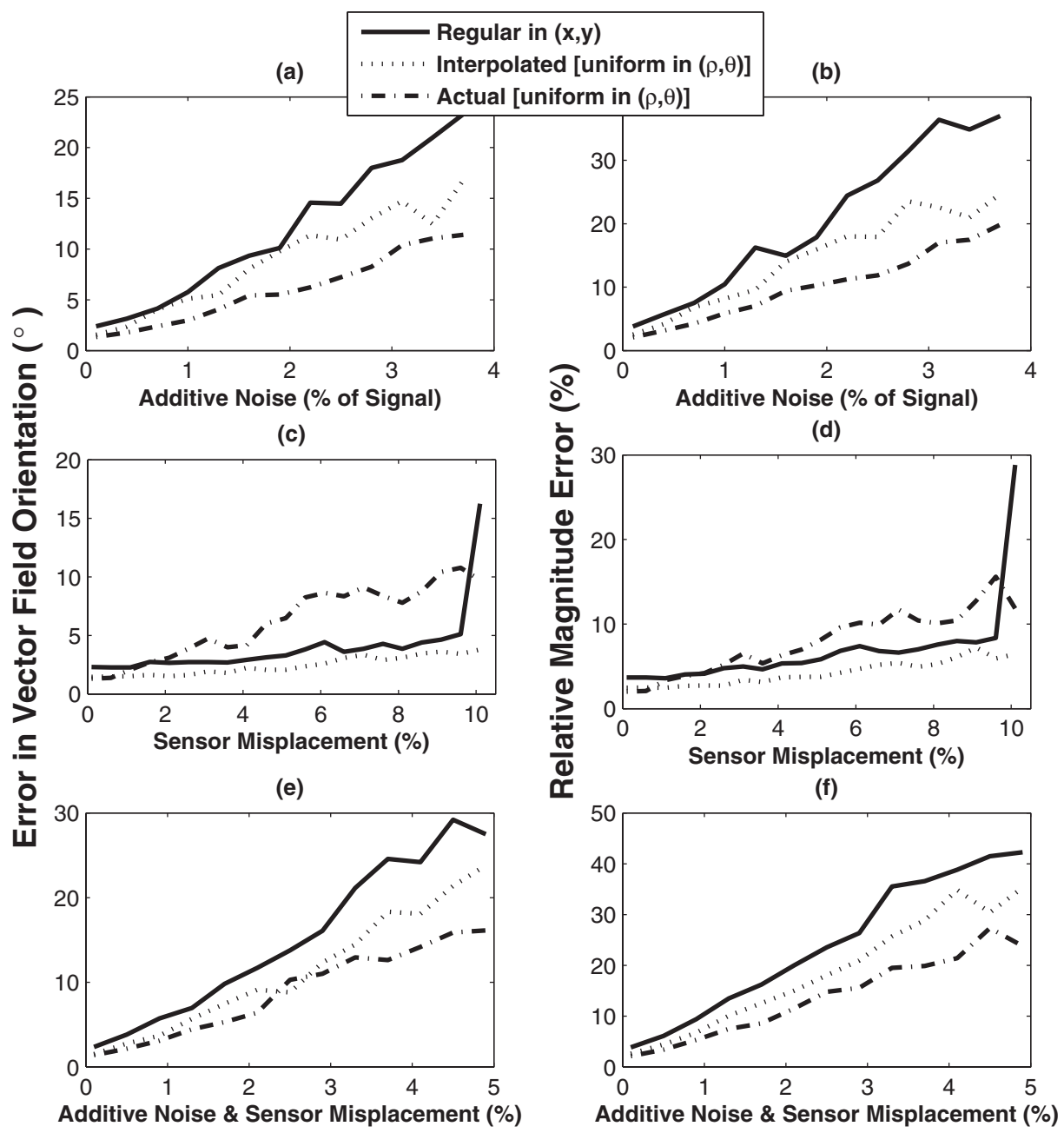

Fig. 9. As in Fig. 7, but $75 \%$ of the sensors were perturbed.

3. 1-D piecewise cubic Hermite interpolation: A cubic Hermite spline is also a third-degree spline. For interpolation on a set of points $\left(x_{i}, S\left(x_{i}\right)\right)(i=1, \ldots, N)$, interpolation is performed in each subinterval $\left(x_{i}, x_{i+1}\right)$ $(i=1, \ldots, N-1)$ by using the formula

$$
\begin{aligned}
S(x)=S_{i}(x)= & (1+2 t)(1-t)^{2} S\left(x_{i}\right)+t(1-t)^{2} h M\left(x_{i}\right) \\
& +t^{2}(3-2 t) S\left(x_{i+1}\right)+t^{2}(t-1) h M\left(x_{i+1}\right),
\end{aligned}
$$

where $t \equiv\left(x-x_{i}\right) / h, h \equiv x_{i+1}-x_{i}$, and $M\left(x_{i}\right)$ and $M\left(x_{i+1}\right)$ are the tangents at $x_{i}$ and $x_{i+1}$, respectively. The choice of tangents is non-unique and there are several options available [31]. Hence, the cubic Hermite spline consists of $N-1$ piecewise cubic Hermite polynomials between the data points.

4. Bilinear interpolation: Assume that we want to find the value of an unknown function $S(x, y)$ at a point $P(x, y)$. It is assumed that we know the values $S_{Q 1}, S_{Q 2}, S_{Q 3}$, and $S_{Q 4}$ of function $S(x, y)$ at the four points $Q 1\left(x_{1}, y_{1}\right)$, $Q 2\left(x_{1}, y_{2}\right), Q 3\left(x_{2}, y_{1}\right)$, and $Q 4\left(x_{2}, y_{2}\right)$, respectively. The desired estimate is given by:

$$
\begin{aligned}
S(x, y)= & \frac{\left(x_{2}-x\right)\left(y_{2}-y\right)}{\left(x_{2}-x_{1}\right)\left(y_{2}-y_{1}\right)} S_{Q 1}+\frac{\left(x_{2}-x\right)\left(y-y_{1}\right)}{\left(x_{2}-x_{1}\right)\left(y_{2}-y_{1}\right)} S_{Q 2} \\
& +\frac{\left(x-x_{1}\right)\left(y_{2}-y\right)}{\left(x_{2}-x_{1}\right)\left(y_{2}-y_{1}\right)} S_{Q 3}+\frac{\left(x-x_{1}\right)\left(y-y_{1}\right)}{\left(x_{2}-x_{1}\right)\left(y_{2}-y_{1}\right)} S_{Q 4} .
\end{aligned}
$$

5. Bicubic interpolation: Assume we have an unknown function $S(x, y)$ that is defined in a rectangle in the $(x, y)$ plane, $a<x<b$ and $c<y<d$. Also, assume that the values of function $S(x, y)$ are known at the grid points $\left(x_{i}, y_{j}\right)(i$ $=1, \ldots, N ; j=1, \ldots, M)$ with $a=x_{1}<x_{2}<\ldots x_{N}=b, \quad c=y_{1}$ $<y_{2}<\ldots y_{M}=d$. The rectangle is decomposed by the grid points into subdomains $R_{i j}$. Subdomain $R_{i j}$ contains the points $(x, y)$ with $x_{i}<x<x_{i+1}, y_{j}<y<y_{j+1}(i=1, \ldots, N-1 ; j$ $=1, \ldots, M-1)$. The $2 \mathrm{D}$ bicubic interpolation scheme is identical to a third-degree polynomial on every $R_{i j}$ :

$$
S(x, y)=S_{i j}(x, y)=\sum_{k=0}^{3} \sum_{l=0}^{3} a_{i j k l}\left(x-x_{i}\right)^{k}\left(y-y_{j}\right)^{l} .
$$

The determination of the $16 M N$ coefficients is performed using the data, the conditions of continuity of the deriva- 


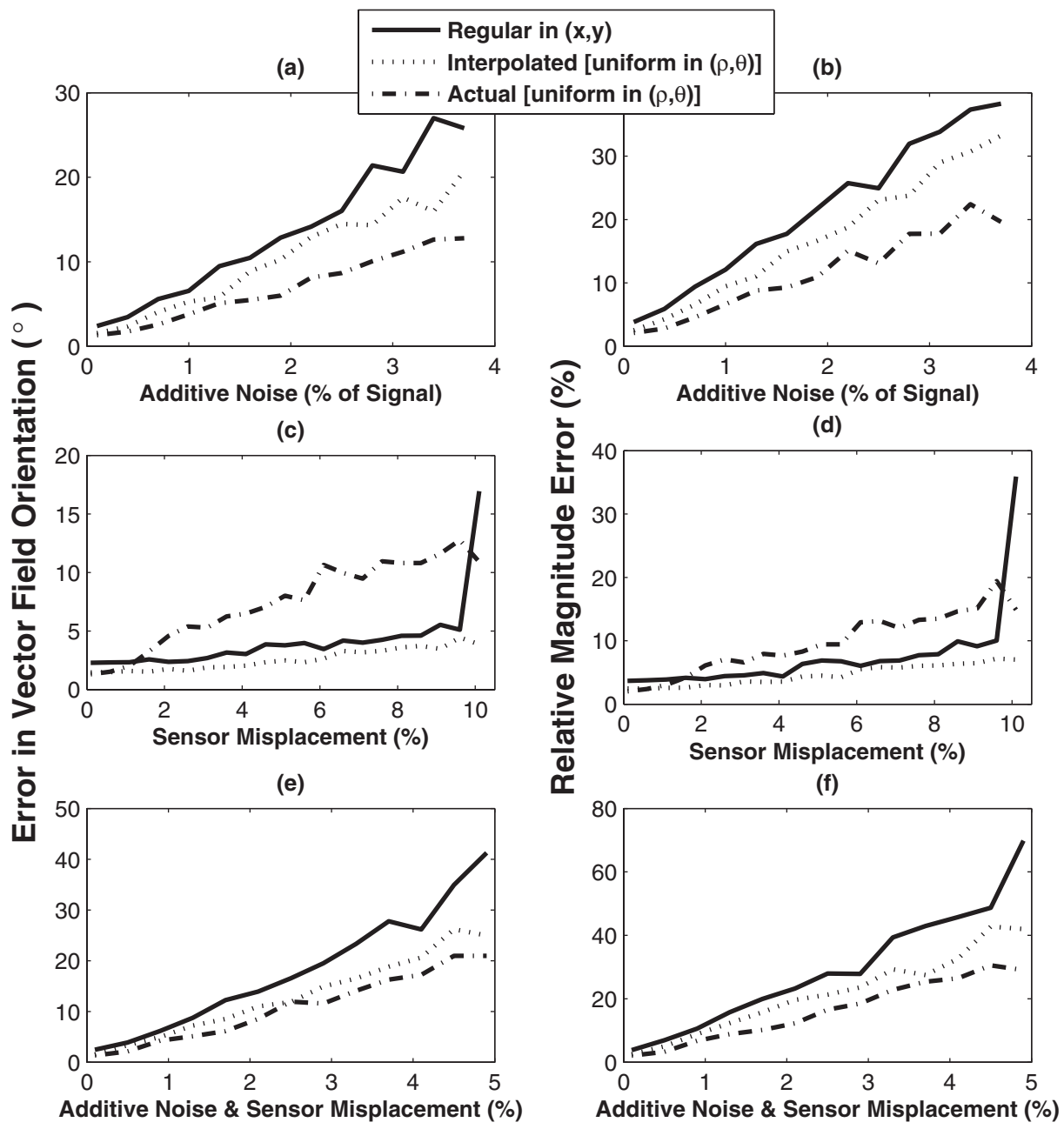

Fig. 10. As in Fig. 7, but all sensors were perturbed.

tives (and cross derivatives) of $S(x, y)$, and some special boundary conditions [31].

6. 2-D spline interpolation: Assume we have the same rectangle in the $(x, y)$ plane $(a<x<b$ and $c<y<d)$, as in the previous interpolation method. Also, assume that the values of function $S(x, y)$ are known at the grid points $\left(x_{i}, y_{j}\right)(i=1, \ldots, N ; j=1, \ldots, M)$ with $a=x_{1}<x_{2}<\ldots x_{N}=b$, $c=y_{1}<y_{2}<\ldots y_{M}=d$. The interpolating spline consists of successive 1-D splines and may be written as

$$
S(x, y)=S_{i j}(x, y)=\sum_{k=1}^{(N-1)+3} \sum_{l=1}^{(M-1)+3} c_{i j k l} u_{i k}(x) v_{j l}(y) .
$$

Coefficients $c_{i j k l}$ are computed by relying on the available data and appropriate algorithms [33]. Also, functions $u_{i k}(x)$ and $v_{j l}(y)$ are chosen so that $\left(u_{1}, \ldots, u_{N+2}\right)$ form a basis of all 1-D, real-valued functions on the bounded interval $[a, b]$ that are piecewise third-degree polynomials on every interval $\left[x_{i}, x_{i+1}\right],(i=1, \ldots, N-1)$. Similarly, functions $\left(v_{1}, \ldots, v_{M+2}\right)$ are selected so that they form a basis of all 1-D, real-valued functions on the bounded interval $[c, d]$ that are piecewise third-degree polynomials on every interval $\left[y_{j}, y_{j+1}\right],(j=1, \ldots, M-1)$ [33]. One such selection yields [33]
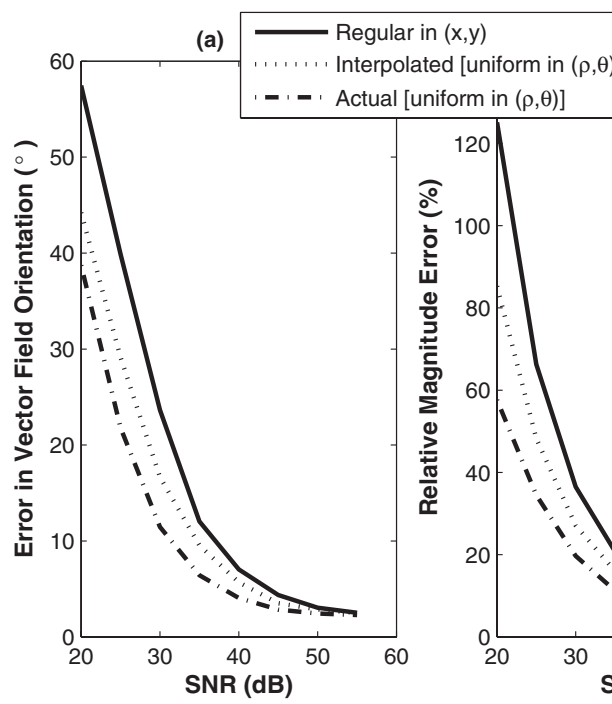

(b)

Fig. 11. Comparison of the reconstruction performance in noisy environments for the cases: (i) when integral data from regularly placed sensors were used; (ii) when interpolated (1-D piecewise cubic spline) measurements that corresponded to uniform sampling with $\Delta \rho=0.5$ and $\Delta \theta=1.5^{\circ}$ were used; and (iii) when actual measurements that corresponded to uniform sampling with $\Delta \rho$ $=0.5$ and $\Delta \theta=1.5^{\circ}$ were used. (a) Error in vector field orientation and (b) error in magnitude when Gaussian noise of zero mean was added to the measurements of the sensors. 


$$
u_{i k}(x)=\Phi\left(\frac{x-a}{h_{1}}+2-i\right), \quad v_{j l}(y)=\Phi\left(\frac{y-c}{h_{2}}+2-j\right)
$$

with

$$
\Phi(t)=\left\{\begin{aligned}
(2-|t|)^{3}, & \text { if } 1<|t|<2 \\
4-6|t|^{2}+3|t|^{3}, & \text { if } 1 \leqslant|1| \\
0, & \text { elsewhere }
\end{aligned}\right.
$$

and $h_{1} \equiv(b-a) /(N-1), h_{2} \equiv(d-c) /(M-1)$.

\section{ACKNOWLEDGMENT}

This work was supported by the Engineering and Physical Sciences Research Council (EPSRC) "Integrated Electronics" portfolio grant.

\section{REFERENCES}

1. S. P. Juhlin, "Doppler tomography," Proceedings of the 15th Annual International Conference of the IEEE Engineering in Medicine and Biology Society, October 28-31, 1993, San Diego, California, USA (1993) pp. 212-213.

2. Y. K. Tao, A. M. Davis, and J. A. Izatt, "Single-pass volumetric bidirectional blood flow imaging spectral domain optical coherence tomography using a modified Hilbert transform," Opt. Express 16,12350-12361(2008).

3. B. M. Howe, P. F. Worcester, and R. C. Spindel, "Ocean acoustic tomography: Mesoscale velocity," J. Geophys. Res. 92, 3785-3806 (1987).

4. W. Munk and C. Wunsch, "Observing the ocean in the 1990s," Philos. Trans. R. Soc. London, Ser. A 307, 439-464 (1982).

5. D. Rouseff, K. B. Winters, and T. E. Ewart, "Reconstruction of oceanic microstructure by tomography: a numerical feasibility study,” J. Geophys. Res. 96, 8823-8833(1991).

6. S. A. Johnson, J. F. Greenleaf, M. Tanaka, and G. Flandro, "Reconstructing three-dimensional temperature and fluid velocity vector fields from acoustic transmission measurements," ISA Trans. 16, 3, pp. 3-15, 1977.

7. D. M. Kramer and P. C. Lauterbur, "On the problem of reconstructing images of non-scalar parameters from projections. Applications to vector fields," ISA Trans. 26, 26742677(1979).

8. S. J. Norton and M. Linzer, "Correcting for ray refraction in velocity and attenuation tomography: a perturbation approach," Ultrason. Imaging 4, 201-233(1982).

9. S. J. Norton, "Tomographic reconstruction of 2-D vector fields: Application to flow imaging," Geophys. J. Int. 97, 161-168 (1988)

10. S. J. Norton, "Unique tomographic reconstruction of vector fields using boundary data," IEEE Trans. Image Process. 1, 406-412 (1992).

11. H. Braun and A. Hauck, "Tomographic reconstruction of vector fields," IEEE Trans. Signal Process. 39, 464471(1991).

12. K. B. Winters and D. Rouseff, "A filtered backprojection method for the tomographic reconstruction of fluid vorticity," Inverse Probl. 6, L33-L38(1990).

13. K. B. Winters and D. Rouseff, "Tomographic reconstruction of stratified fluid flow," IEEE Trans. Ultrason. Ferroelectr. Freq. Control 40, 26-33(1993).
14. M. Zahn, "Transform relationship between Kerr-effect optical phase shift and non-uniform electric field distributions," IEEE Trans. Dielectr. Electr. Insul. 1, 235-246(1994).

15. H. M. Hertz, "Kerr effect tomography for nonintrusive spatially resolved measurements of asymmetric electric field distributions," Appl. Opt. 25, 914-921(1986).

16. H. K. Aben, "Kerr effect tomography for general axisymmetric field," Appl. Opt. 26, 2921-2924(1987).

17. N. P. Efremov, N. P. Poluektov, and V. N. Kharchenko, "Tomography of ion and atom velocities in plasmas," J. Quant. Spectrosc. Radiat. Transf. 53, 723-728(1995).

18. G. W. Faris and R. L. Byer, "Three-dimensional beamdeflection optical tomography of a supersonic jet," Appl. Opt. 27, 5202-5212(1988).

19. V. A. Sharafutdinov, "Tomographic problem of photoelasticity," Proc. SPIE 1843, 234-243(1992).

20. H. Aben and A. Puro, "Photoelastic tomography for threedimensional flow birefringence studies," Inverse Probl. 13, 215-221(1997).

21. A. Schwarz, "Three-dimensional reconstruction of temperature and velocity fields in a furnace," Proceedings of ECAPT, The European Concerted Action on Process Tomography (International Society for Industrial Process Tomography, 1994), pp. 227-233.

22. S. E. Segre, "The measurement of poloidal magnetic field in a tokamak by the change of polarization of an electromagnetic wave," Plasma Phys. 20, 295-307(1978).

23. P. Juhlin, "Principles of Doppler Tomography," Lund Institute of Technology, Sweden, Department of Mathematics, LUTFD2/(TFMA-92)/7002+17P, August (1992).

24. M. Petrou and A. Giannakidis, "Complete tomographic reconstruction of 2 -d vector fields using a system of linear equations," Proceedings of the 12th Annual Medical Image Understanding and Analysis Conference (MIUA 2008) July 2-3, 2008, Dundee, Scotland, UK, pp. 132-136.

25. S. R. Deans, The Radon Transform and Some of Its Applications (Wiley, 1983).

26. M. Petrou and A. Kadyrov, "Affine invariant features from the trace transform," IEEE Trans. Pattern Anal. Mach. Intell. 26, 30-44(2004).

27. A. Kadyrov and M. Petrou, "Affine parameter estimation from the trace transform," IEEE Trans. Pattern Anal Mach. Intell. 28, 1631-1645(2006).

28. D. Kincaid and W. Cheney, Numerical Analysis: Mathematics of Scientific Computing(American Mathematical Society, 2002).

29. H. R. Schwarz, Numerische Mathematik (B. G. Teubner, 1986).

30. H. Schwetlick and H. Kretzschmar, Numerische Verfahren fur Naturwissenschaftler und Ingenieure (Fachbuchverlag, 1991).

31. I. N. Bronshtein, K. A. Semendyayev, G. Musiol, and H. Muehlig, Handbook of Mathematics (Springer, 2003).

32. C. de Boor, A Practical Guide to Splines (Springer-Verlag, 1978).

33. C. Habermann and F. Kindermann, "Multidimensional spline interpolation: Theory and applications," Comput. Econ. 30, 153-169(2007).

34. R. M. Rangayyan, M. Ciuc, and F. Faghih, "Adaptedneighborhood filtering of images corrupted by signaldependent noise," Appl. Opt. 37, 4477-4487(1998).

35. T. D. Sanger, J. Kaiser, and B. Placek, "Reaching movements in childhood dystonia contain signal-dependent noise," J. Child Neurol. 20, 489-496(2005).

36. G. Krüger and G. H. Glover, "Physiological noise in oxygenation-sensitive magnetic resonance imaging," Magn. Reson. Med. 46, 631-677(2001). 